\title{
The many metabolic sources of acetyl-CoA to support histone acetylation and influence cancer progression
}

\section{Olivier Feron}

Pole of Pharmacology and Therapeutics (FATH), Institut de Recherche Expérimentale et Clinique (IREC), UCLouvain, Brussels, Belgium Correspondence to: Prof. Olivier Feron. Pole of Pharmacology and Therapeutics (FATH), Institut de Recherche Expérimentale et Clinique (IREC), UCLouvain, 57 avenue Hippocrate, B1.57.04, B-1200 Brussels, Belgium. Email: olivier.feron@uclouvain.be.

Provenance: This is an invited article commissioned by the Section Editor Dr. Ran Mo (Department of Burn and Plastic Surgery, Nanjing Drum Tower Hospital, Nanjing, China).

Comment on: Sun RC, Dukhande VV, Zhou Z, et al. Nuclear glycogenolysis modulates histone acetylation in human non-small cell lung cancers. Cell Metab 2019;30:903-16.e7.

Submitted Nov 11, 2019. Accepted for publication Nov 25, 2019.

doi: 10.21037/atm.2019.11.140

View this article at: http://dx.doi.org/10.21037/atm.2019.11.140

The fundamental unit of chromatin is the nucleosome which is composed of a histone octamer and the DNA that wraps around it. Histones are globular proteins subject to various reversible covalent modifications that primarily occur on their flexible $\mathrm{N}$-terminal ends (the so-called tail). Histone acetylation is one of these major alterations that may influence the chromatin conformation and consecutively influence gene expression (1). Histone acetylation is usually associated with an increase in transcriptional activity. Indeed, since acetylation occurs on positively charged lysines residues, the addition of an acetyl group on these residues changes the overall charge of the histone tail thereby leading to weaker binding of the nucleosomal components (2). As a direct consequence of histone acetylation, DNA becomes more accessible to transcription factors. Enzymes named histone acetyltransferases (HATs) catalyzed this mode of posttranslational modifications (3). Other enzymes termed histone deacetylases (HDACs) are involved in the reverse process of histone deacetylation that restores the ionic interactions between positively charged histones and negatively charged DNA, thereby yielding a more compact chromatin structure (making it harder for transcription factors to bind to the DNA) $(2,3)$.

The extent of histone acetylation is thus regulated by changes in the expression and activity of HAT and HDAC enzymes. In the field of cancer, it is often proposed that increase in histone acetylation supports tumor progression by promoting aberrant transcription of key genes regulating important cellular functions such as cell proliferation and cell-cycle regulation (4). The nature of the genes that are regulated upon histone (de)acetylation may however tip the balance in opposite directions. Tumor progression can for instance be promoted if a tumor suppressor gene is silenced upon a decrease in histone acetylation resulting from HDAC high expression or aberrant recruitment to an inadequate locus (5). This caveat complicates the task of developing drugs acting as epigenetic modulators to block cancer progression (6). The pan-inhibitory nature of epigenetic modifiers indeed accounts for a lack of selectivity toward the specific target (that is primarily causing the alteration) and thus also for adverse effects resulting from effects on physiological epigenetic pathways.

With the renewed interest for the study of cancer metabolism, another key parameter in the regulation of histone acetylation in tumors has emerged, namely the extent of available nuclear acetyl coenzyme-A (acetyl-CoA), i.e., the substrate that HATs use to transfer an acetyl group onto a histone lysine. Besides the historically well-studied glucose as a major source of acetyl-CoA, several reports have documented in the last few years that other metabolic fuels including glutamine, fatty acids (FA), acetate and even lactate, could represent alternate sources of acetyl-CoA and thus potentially influence the histone (de-)acetylation processes (7). In their recent publication in Cell Metabolism, Sun and colleagues (8) have identified nuclear glycogen as 

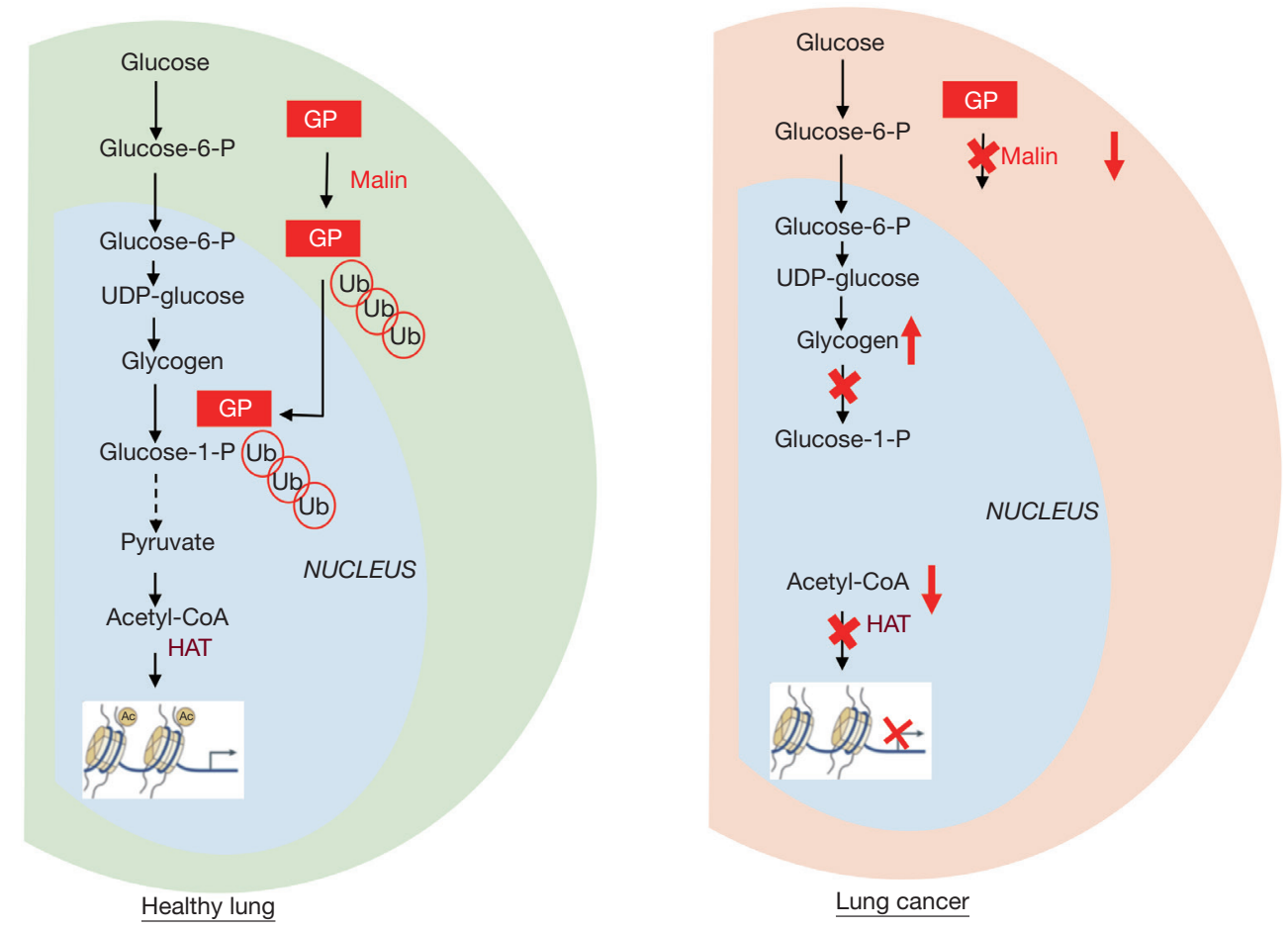

Figure 1 Loss of malin in NSCLC accounts for a reduced histone acetylation and tumor progression. Malin is a RINGtype E3 ubiquitin ligase that promotes the ubiquitination and consecutive nuclear translocation of glycogen phosphorylase (GP) into the nucleus. GP catalyses the rate-limiting step in glycogenolysis by releasing glucose-1-phosphate (that after conversion in glucose-6-phosphate feeds glycolysis). In healthy lung, de novo synthesized nuclear glycogen provides, upon GP activity, a carbon pool for histone acetylation. In NSCLC, the loss of malin prevents nuclear glycogenolysis, in fine reducing histone acetylation and promoting tumor growth. NSCLC, non-small cell lung cancer.

an additional carbon pool for histone acetylation (Figure 1). Since glycogen is a well-known source of glucose-6phosphate (i.e., a glycolytic precursor of pyruvate and acetyl-CoA), the bearing of this discovery may look quite limited at a first glance. This is however just the opposite. Indeed, these authors have demonstrated that in order for nuclear glycogen to contribute to a pyruvate pool for histone acetylation, glycogen synthesis has to occur de novo in the nucleus. They further identified the ubiquitination of an abundant isoform of glycogen phosphorylase (GP) by the E3 ubiquitin ligase malin as the main trigger of the necessary glycogenolysis that in fine provides the substrate for histone acetylation (Figure 1). In other words, ubiquitination of GP by malin does not favor its proteasomal degradation but instead supports its trafficking from the cytoplasm to the nucleus where pyruvate can be generated from glucose-6P (via the action of nuclear glycolytic enzymes) and gives rise to acetyl-CoA to support histone modification (Figure 1).

There are potential therapeutic implications of this finding since the above malin-driven pathway is altered in human non-small cell lung cancers (NSCLC) (Figure 1) (8). The investigators at the University of Kentucky identified in lung cancer cell lines and clinical lung specimens, a decreased abundance of malin that was associated with an impaired translocation of GP and thus a deficiency in nuclear glycogenolysis (Figure 1). They have also documented that malin-deficient bronchioalveolar stem cells yielded a significantly higher amount of lung organoids than wild-type stem cells. The demonstration is further strengthened by evidence that nuclear glycogen accumulated in lung cancer cells (Figure 1) and that re-introduction of malin in these cells restored nuclear glycogenolysis, increased histone acetylation and decreased growth of tumor xenografts in mice. Although the authors concluded that the nuclear glycogen metabolic pathway represents 
a novel therapeutic target for NSCLC, it is difficult to anticipate whether such global interference with glycogendependent epigenetic regulations in cancer cells but also healthy cells could clinically translate in an efficient and safe therapeutic modality. The rationale for developing drugs to promote nuclear glycogenolysis is actually at the opposite of the current evaluation of GP inhibitors to prevent the use of glycogen as energy source by cancer cells and thereby to block the formation of metastases (9). Still, in NSCLC, the beneficial effects of promoting histone acetylation (instead of blocking it) is supported by encouraging results obtained with HDAC inhibitors such as vorinostat or suberoylanilide hydroxamic acid (SAHA) (10,11). Moreover, the option of blocking GP may expose cancer patients to various adverse effects since GP exists as different tissue isoforms exhibiting up to $80 \%$ homology (12). Blocking GP activity may for instance interfere with ATP generation required for muscle contraction but also with the capacity of the central nervous system to handle periods of severe hypoglycemia. Astrocytic glycogen-derived lactate is indeed known to maintain neuronal functions such as memory formation but also to maintain brain ATP levels during exhaustive exercise (13). Potential neurological adverse effects may thus derive from the use of poorly selective GP inhibitors. Today the field of GP inhibition is mainly driven by the search for tissue-specific GP inhibitors, in particular for drugs able to block the liver GP isoform since the consecutive hepatic stimulation of glycogen synthesis makes this type of drug particularly suited for the treatment of type 2 diabetes (14). To reach the clinics, this new generation of antidiabetic drugs will have to spare the other GP isoforms, in particular in the brain since GP inhibition in the skeletal muscle somehow also contributes to glucose clearance from blood. Of note, blocking muscle GP may however not be harmless for diabetic patients since it may alter their capacity to undertake aerobic exercise training that is known to be critical to slow down the progression of the disease (15). Because of the anticipated difficulty related to unspecific inhibition of GP isoforms, stimulating GP as supported by the data of Sun et al. (8) may well represent a more relevant anticancer strategy, in particular to tackle NSCLC. Studies are thus warranted to identify biomarkers able to determine which epigenetic landscape is the most likely to take advantage of this therapeutic approach that increases or restore nuclear acetylation.

Modulating histone acetylation through manipulation of the tumor metabolism also requires to integrate the contributions of various pathways to the cellular and nuclear acetyl-CoA pool. Indeed, as acknowledged by Sun and colleagues, their elegant experimental approaches including state-of-the-art nucleus isolation to prove the occurrence of nuclear glycogenolysis, do not offer insights on the extent of pyruvate generated in the cytosol and subsequently transported into the nucleus to generate acetyl-CoA. Yet more striking, acetate, glutamine and FA can generate acetyl-CoA in a glucose-independent manner and thus also influence the extent of histone acetylation in cancer cells (Figure 2) $(7,16,17)$. Acetyl-CoA may for instance be (re-)generated from citrate or acetate, upon the activity of ATP-citrate lyase (ACLY) $(18,19)$ and acylCoA synthetase short-chain family member (ACSS) $(20,21)$, respectively (Figure 2). Of note, glucose- or lactate-derived pyruvate is metabolized to acetyl-CoA by the pyruvate dehydrogenase complex (PDC) in the mitochondria but since acetyl-CoA cannot freely cross the organelle membrane, it is translocated to the cytosol in the form of either citrate (formed upon condensation with oxaloacetate by citrate synthase) or acetylcarnitine (upon conversion by carnitine acetyltransferase) (22) (Figure 2). Citrate may also derive from glutamine, including through reductive carboxylation (23). Among the other major sources of acetyl-CoA are the FA upon oxidation in the mitochondria (Figure 2) (24). FA were actually reported as the predominant contributor to the acetylation of certain histone lysines (even in the presence of excess glucose) in an ACLY-independent manner (25). It remains however unclear how mitochondrial FA-derived acetyl-CoA is translocated into the nucleus and to which extent the length and unsaturation of the FA chain influence this process (26).

The plethora of pathways possibly fueling the acetylCoA pool in cancer cells and thus potentially regulating histone acetylation explains the intricate relationship between metabolism and gene transcription reported in various recent studies. In pancreatic ductal adenocarcinoma (PDA) cells, Kras-driven ACLY signaling was documented to support histone acetylation in response to increase in acetyl-CoA abundance, thereby facilitating cell plasticity and proliferation (27). Another example of the interplay between bioenergetics and histone acetylation arises from the demonstration that the tumor acidic microenvironment is associated with sirtuin-mediated histone deacetylation and consecutive silencing of acetyl-CoA carboxylase ACC2, making mitochondrial fatty acyl-CoA degradation compatible with cytosolic lipogenesis $(28,29)$. Interestingly, the metabolic source of acetyl-CoA somehow seems to dictate which transcriptional program will be favored. 


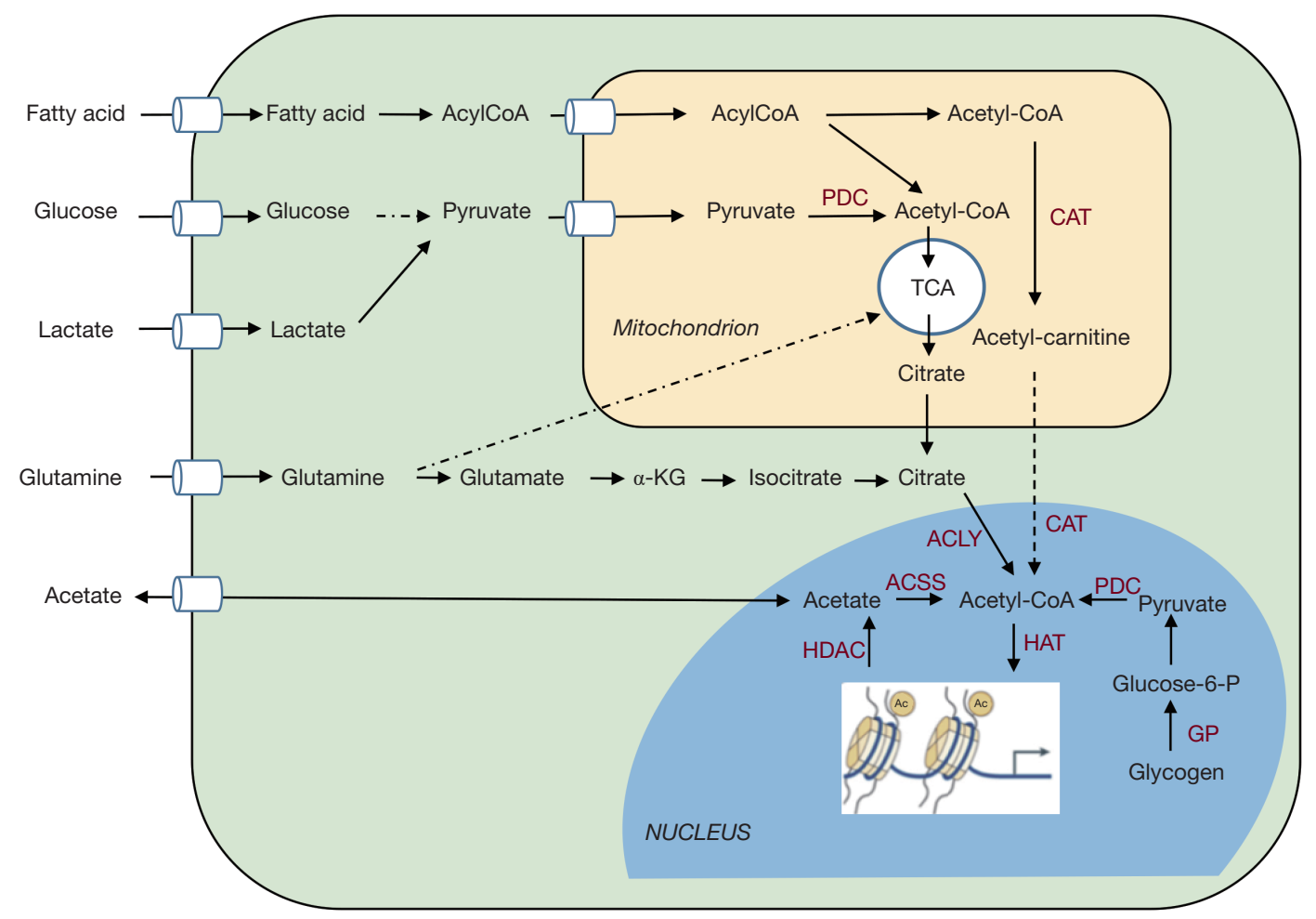

Figure 2 Acetyl-CoA production and utilization for histone acetylation. Glucose, fatty acids, and acetate but also glutamine and lactate can contribute to acetyl-CoA production. PDC convert pyruvate (arising either from glucose or lactate) into acetyl-CoA in the mitochondria. Transport of acetyl-CoA produced in mitochondria to the cytosol and consecutively to the nucleus relies on citrate export and cleavage by ACLY. An alternate path, also possibly used for acetyl-CoA resulting from fatty acid oxidation (and not directly used in the TCA cycle), is the conversion to acetylcarnitine by CAT and the consecutive transport by mitochondrial translocase. As for acetate captured from outside the cell or resulting from intracellular protein deacetylation, it does not enter into mitochondria and is converted into acetyl-CoA via the activity of ACSS. The balance between the activity of ACLY and ACSS is partly influenced by the availability in nutrients and $\mathrm{O}_{2}$, ACCS2 being particularly active under metabolic stress. Glutamine can also give rise to acetyl-CoA from citrate produced either in mitochondria through the conventional TCA cycle or in the cytosol upon reductive carboxylation. ACLY, ACSS (as a specific isoform, ACSS2) and CAT are expressed in the nucleus, directly delivering acetyl-CoA for histone modification and consecutive regulation of gene transcription. Cytosolic pyruvate (not depicted) and nuclear-generated pyruvate derived from glycogenolysis through the activity of GP may also represent an important source of acetyl-CoA after conversion by PDC located into the nucleus. ACLY, ATP-citrate lyase; ACSS, Acyl-CoA synthetase short-chain; PDC, pyruvate dehydrogenase complex; CAT, carnitine-acetyltransferase; TCA cycle, tricarboxylic acid cycle; GP, glycogen phosphorylase; HAT, histone acetyltransferase; HDAC, histone deacetylase.

For instance, FA-dependent histone acetylation drives the activation of lipid oxidation-specific genes (25) while high-glucose conditions promote the hyperacetylation of histones, which in turn favors the expression of the glucose transporter GLUT4 and major glycolytic enzymes (18).

Finally, it should be stressed that histone acetylation cannot be dissociated from another histone modification, namely histone methylation that can recruit different regulators of gene transcription (30). In particular, methylation of histone at lysines 9, 27, and 20 (H3K9, $\mathrm{H} 3 \mathrm{~K} 27$, and H4K20) is often associated with repressed transcription, whereas methylation of $\mathrm{H} 3 \mathrm{~K} 4, \mathrm{H} 3 \mathrm{~K} 36$, and H3K79 correlates with transcriptionally active euchromatin. As for histone (de)acetylation, enzymes that influence histone methylation utilize co-substrates or co-factors generated by cellular metabolism, thereby providing an additional link between epigenetic and cancer metabolism $(31,32)$. The list of potential metabolic modulators of 
epigenetic enzymes includes acetyl and methyl donor groups such as acetyl-CoA and S-adenosylmethionine, respectively, but also oxygen, ATP, NAD, FAD, and various TCA cycle intermediates such as 2-oxoglutarate, succinate and fumarate (33) and will certainly keep expanding in the future.

In conclusion, histone acetylation is intimately coordinated with cellular acetyl-CoA pool, itself reflecting the oncogenic metabolic preferences and/or the nutrient availability. Even glycogen, the primary source of longterm sugar storage in mammals contributes to histone acetylation upon nuclear breakdown into pyruvate. While this is normally kept under check in the lung, a deficit in nuclear GP directly impacts on the histone modification in NSCLC, thereby participating to tumor progression. Altogether these findings support a paradigm shift in our understanding of tumor growth. Indeed, while tumor metabolism has been most often considered as a consequence of oncogenic signals to support cancer cell growth, it is nowadays clear that gene expression/repression associated with cancer progression may directly depend on the availability of nutrients arising from both the exogenous milieu and internal stores.

\section{Acknowledgments}

Funding: The experimental work of the author is supported by grants from the Fonds de la Recherche Scientifique (F.R.S.-FNRS), the Télévie, the Belgian Foundation against cancer, the J. Maisin Foundation and an Action de Recherche Concertée de la Communauté française de Belgique (ARC 19/24-096).

\section{Footnote}

Conflicts of Interest: The author has no conflicts of interest to declare.

Ethical Statement: The author is accountable for all aspects of the work in ensuring that questions related to the accuracy or integrity of any part of the work are appropriately investigated and resolved.

\section{References}

1. Tessarz P, Kouzarides T. Histone core modifications regulating nucleosome structure and dynamics. Nat Rev Mol Cell Biol 2014;15:703-8.
2. Barnes CE, English DM, Cowley SM. Acetylation \& Co: an expanding repertoire of histone acylations regulates chromatin and transcription. Essays Biochem 2019;63:97-107.

3. Verdin E, Ott M. 50 years of protein acetylation: from gene regulation to epigenetics, metabolism and beyond. Nat Rev Mol Cell Biol 2015;16:258-64.

4. Cai L, Sutter BM, Li B, et al. Acetyl-CoA induces cell growth and proliferation by promoting the acetylation of histones at growth genes. Mol Cell 2011;42:426-37.

5. Di Cerbo V, Schneider R. Cancers with wrong HATs: the impact of acetylation. Brief Funct Genomics 2013;12:231-43.

6. Wapenaar H, Dekker FJ. Histone acetyltransferases: challenges in targeting bi-substrate enzymes. Clin Epigenetics 2016;8:59.

7. Corbet C, Feron O. Cancer cell metabolism and mitochondria: Nutrient plasticity for TCA cycle fueling. Biochim Biophys Acta Rev Cancer 2017;1868:7-15.

8. Sun RC, Dukhande VV, Zhou Z, et al. Nuclear Glycogenolysis Modulates Histone Acetylation in Human Non-Small Cell Lung Cancers. Cell Metab 2019;30:903-16.e7.

9. Curtis M, Kenny HA, Ashcroft B, et al. Fibroblasts Mobilize Tumor Cell Glycogen to Promote Proliferation and Metastasis. Cell Metab 2019;29:141-55.e9.

10. Reguart N, Rosell R, Cardenal F, et al. Phase I/II trial of vorinostat (SAHA) and erlotinib for non-small cell lung cancer (NSCLC) patients with epidermal growth factor receptor (EGFR) mutations after erlotinib progression. Lung Cancer 2014;84:161-7.

11. Gray JE, Saltos A, Tanvetyanon T, et al. Phase I/Ib Study of Pembrolizumab Plus Vorinostat in Advanced/ Metastatic Non-Small Cell Lung Cancer. Clin Cancer Res 2019;25:6623-32.

12. Mathieu C, Dupret JM, Rodrigues Lima F. The structure of brain glycogen phosphorylase-from allosteric regulation mechanisms to clinical perspectives. FEBS J 2017;284:546-54.

13. Matsui T, Omuro H, Liu YF, et al. Astrocytic glycogenderived lactate fuels the brain during exhaustive exercise to maintain endurance capacity. Proc Natl Acad Sci U S A 2017;114:6358-63.

14. Rath VL, Ammirati M, LeMotte PK, et al. Activation of human liver glycogen phosphorylase by alteration of the secondary structure and packing of the catalytic core. Mol Cell 2000;6:139-48.

15. Baker DJ, Greenhaff PL, MacInnes A, et al. The 
experimental type 2 diabetes therapy glycogen phosphorylase inhibition can impair aerobic muscle function during prolonged contraction. Diabetes 2006;55:1855-61.

16. Corbet C, Feron O. Metabolic and mind shifts: from glucose to glutamine and acetate addictions in cancer. Curr Opin Clin Nutr Metab Care 2015;18:346-53.

17. Pietrocola F, Galluzzi L, Bravo-San Pedro JM, et al. Acetyl coenzyme A: a central metabolite and second messenger. Cell Metab 2015;21:805-21.

18. Wellen KE, Hatzivassiliou G, Sachdeva UM, et al. ATP-citrate lyase links cellular metabolism to histone acetylation. Science 2009;324:1076-80.

19. Lee JV, Carrer A, Shah S, et al. Akt-dependent metabolic reprogramming regulates tumor cell histone acetylation. Cell Metab 2014;20:306-19.

20. Gao X, Lin SH, Ren F, et al. Acetate functions as an epigenetic metabolite to promote lipid synthesis under hypoxia. Nat Commun 2016;7:11960.

21. Schug ZT, Peck B, Jones DT, et al. Acetyl-CoA synthetase 2 promotes acetate utilization and maintains cancer cell growth under metabolic stress. Cancer Cell 2015;27:57-71.

22. Madiraju P, Pande SV, Prentki M, et al. Mitochondrial acetylcarnitine provides acetyl groups for nuclear histone acetylation. Epigenetics 2009;4:399-403.

23. Mullen AR, Wheaton WW, Jin ES, et al. Reductive carboxylation supports growth in tumour cells with defective mitochondria. Nature 2011;481:385-8.

Cite this article as: Feron O. The many metabolic sources of acetyl-CoA to support histone acetylation and influence cancer progression. Ann Transl Med 2019;7(Suppl 8):S277. doi: 10.21037/atm.2019.11.140
24. Corbet C, Feron O. Emerging roles of lipid metabolism in cancer progression. Curr Opin Clin Nutr Metab Care 2017;20:254-60.

25. McDonnell E, Crown SB, Fox DB, et al. Lipids Reprogram Metabolism to Become a Major Carbon Source for Histone Acetylation. Cell Rep 2016;17:1463-72.

26. Dierge E, Feron O. Dealing with saturated and unsaturated fatty acid metabolism for anticancer therapy. Curr Opin Clin Nutr Metab Care 2019;22:427-33.

27. Carrer A, Trefely S, Zhao S, et al. Acetyl-CoA Metabolism Supports Multistep Pancreatic Tumorigenesis. Cancer Discov 2019;9:416-35.

28. Corbet C, Draoui N, Polet F, et al. The SIRT1/HIF2alpha axis drives reductive glutamine metabolism under chronic acidosis and alters tumor response to therapy. Cancer Res 2014;74:5507-19.

29. Corbet C, Pinto A, Martherus R, et al. Acidosis Drives the Reprogramming of Fatty Acid Metabolism in Cancer Cells through Changes in Mitochondrial and Histone Acetylation. Cell Metab 2016;24:311-23.

30. Greer EL, Shi Y. Histone methylation: a dynamic mark in health, disease and inheritance. Nat Rev Genet 2012;13:343-57.

31. Dawson MA, Kouzarides T. Cancer epigenetics: from mechanism to therapy. Cell 2012;150:12-27.

32. Lu C, Thompson CB. Metabolic regulation of epigenetics. Cell Metab 2012;16:9-17.

33. Kaelin WG Jr, McKnight SL. Influence of metabolism on epigenetics and disease. Cell 2013;153:56-69. 